\title{
Experimental Characterization of the Effective Propagation Constant of Dense Random Media
}

\author{
Adib Nashashibi, Member, IEEE, and Kamal Sarabandi, Senior Member, IEEE
}

\begin{abstract}
In this paper, a new technique for measuring the effective propagation constant of dense random media is presented. This technique involves two major steps: 1) measurement of the mean bistatic scattered field of a cluster of the random medium confined in a spherical boundary and 2) characterization of the complex permittivity for a homogeneous dielectric sphere having identical radius and bistatic scattered field as those of the spherical cluster of the random medium. Using this measurement technique, not only the effective propagation constant of complex dense random media for which analytical solution does not exist can be characterized, but it can also be used to establish the validity region of the existing models. The sensitivity analyses of the proposed algorithm show that the imaginary part of the effective propagation constant can be measured very accurately. It is also shown that the effective complex permittivity of media with very low dielectric contrast or volume fractions can be characterized accurately. Measurements of the effective propagation constant of different dense random media comprised of homogeneous spherical particles of different packing densities are reported and compared with the existing analytical models.
\end{abstract}

Index Terms - Electromagnetic propagation in nonhomogeneous media.

\section{INTRODUCTION}

C HARACTERIZATION of the propagation constant of the mean field in the random medium is of great importance for modeling electromagnetic wave propagation and scattering in random media. There are a number of analytical models for the calculation of the effective propagation constant in random media. Among these, the most widely used models are: the Polder-Van Santen mixing formula [1], the effective field approximation (EFA), and the quasi-crystalline approximation (QCA) [2]. However, the validity of these models in characterizing the effective propagation constant of dense random media has not been determined, except at low particle volume fractions [6]. The literature concerning experimental characterization of the effective propagation constant $K$ of random media is rather scarce. In general, measurement techniques can be categorized into low- and high-frequency approaches. In low frequencies when the particle dimensions are much smaller than the wavelength, standard dielectric measurement techniques can be used to measure $K$ [3]-[5]. At high frequencies, the standard approach is the free-space

Manuscript received August 15, 1995; revised March 8, 1996.

The authors are with the Radiation Laboratory, Department of Electrical Engineering and Computer Science, University of Michigan, Ann Arbor, MI 48109 USA.

Publisher Item Identifier S 0018-926X(99)07056-8. transmission measurement technique (FSTM) [6], [7]. In this technique, a slab of the dense random medium is positioned between two antennas and the transmission coefficient is measured coherently. Then by moving the slab and measuring the transmission coefficient repeatedly, the mean transmission coefficient is computed from which the effective propagation constant of the random medium is calculated. Although the measurement of the effective propagation constant using the FSTM technique is simple in principle, its accuracy is limited by a number of practical difficulties including: 1) maintaining the phase coherency between the receiver and the transmitter during measurements because of RF cables movement and 2) large slab size. Another drawback of the FSTM method stems from the fact that the effective propagation constant must be computed from a single measured parameter of the medium, namely, the mean transmission coefficient. Hence, the accuracy of the measurement of $K$ is severely limited by the systematic errors. Also, the accuracy in the evaluation of $K$ using this method decreases for dense random media with significant extinction rates.

Mandt $e t$ al. used the FSTM method [6] to measure $K$ of dense random media (volume fraction $f \leq 0.1$ ) and compared the measured extinction coefficient with those predicted by EFA and QCA. At these volume fractions, the analytical models were in good agreement with the measured extinctions. This is also confirmed by the results which will be reported in this paper. However, due to the limitations inherent in the FSTM technique, it was difficult to measure the effective propagation constant of dense media with higher volume fractions.

In this paper, a novel approach for the measurement of the effective propagation constant of dense random media is presented which circumvents most of the aforementioned difficulties of the standard approach. Using the new approach, it is shown that the effective propagation constant of random media can be measured very accurately over a wide range of volume fractions, thereby establishing a benchmark that can be used for the characterization of the validity regions of the existing analytical models. Besides the benchmarking purpose, the measurement technique may be used for the development of empirical models for the effective propagation constant of random media of interest. In the following sections, first the new measurement technique is described and practical issues such as measurement accuracy and sensitivity analysis are discussed. In Section III, the validity of the measurement 


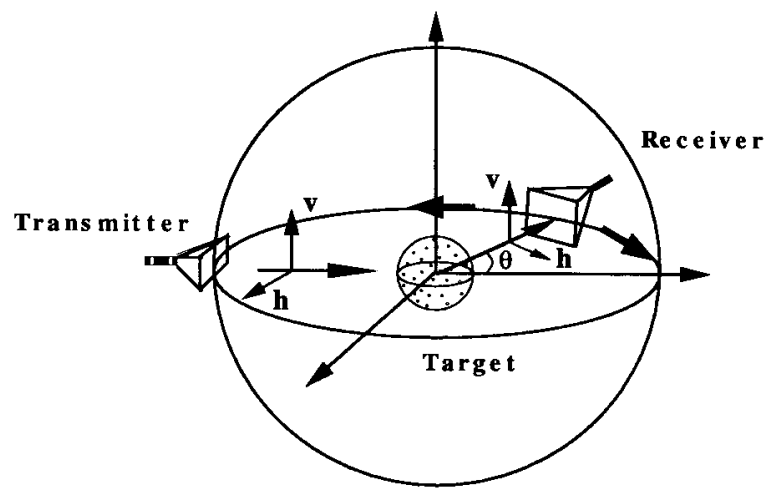

(a)

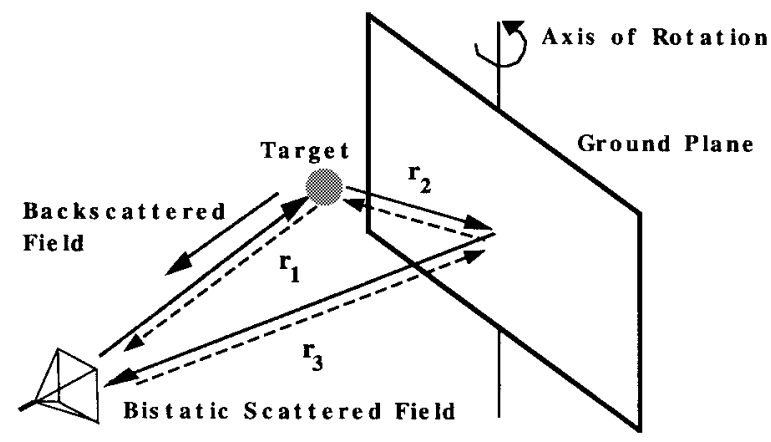

(b)

Fig. 1. The proposed measurement setup. (a) Bistatic radar measuring a cluster of a random medium. (b) Monostatic radar in conjunction with a rotatable ground plane measuring the bistatic response of a cluster of a random medium.

technique is demonstrated by performing experiments on random media for which the effective propagation constant is known. In Section IV, measurements of dense random media of different volume fractions are presented and compared with existing analytical models.

\section{COHERENT Bistatic SCATTERING MEASUREMENT TECHNIQUE (CBSM)}

In this section, a new measurement technique for characterizing the effective propagation constant of a dense random medium is presented. First, a cluster of constituent particles of the random medium is confined in a rotationally symmetric geometry such as a sphere or a cylinder. Then, the copolarized bistatic scattering amplitudes $S_{v v}$ and $S_{h h}$ of the cluster are measured over many bistatic scattering angles $(\theta)$ in a single scattering plane, as shown in Fig. 1(a). At each bistatic angle, the radar measurements are performed over many independent realizations of the random medium from which the coherent and incoherent components of the scattered amplitudes are computed. Independent bistatic measurements for a given bistatic angle can be realized by simply rotating the cluster of the random medium about its axis of symmetry and/or randomizing the particles within the boundary. To evaluate the effective propagation constant of the dense random medium, the measured mean bistatic scattering amplitudes are fitted to the bistatic scattering pattern of a homogeneous dielectric material having the same geometrical boundary. Toward this end, a constrained search routine [8], can be used to minimize the difference between the measured and theoretical responses. With the real and imaginary parts of $K$ acting as free parameters, the following error function is minimized as shown in (1) at the bottom of the page, where the superscripts $m$ and $t$ refer to the measured and theoretical responses, respectively, $N$ refers to the total number of bistatic angles measured, and $M$ refers to the total number of frequency points measured. Quantities $s$ and $\phi$ refer to the magnitude and phase of the copolarized mean bistatic scattering amplitudes, respectively. The CBSM technique offers a number of advantages over the FSTM technique in that measurements need only be performed on a small volume of the random medium and that more than one measured parameter is used to characterize $K$. Basically, the scattering properties of a cluster of the random medium can be accurately characterized in an anechoic chamber environment. In addition, it is rather convenient to position and orient a relatively small target (the cluster of the random medium) and, therefore, it becomes possible to measure many independent samples of the random media independent of their volume fractions. Moreover, since in characterization of the effective propagation constant, bistatic measurements at many bistatic angles are used the effect of systematic errors on the overall measurement accuracy is minimized.

It should be noted here that a phase-coherent polarimetric bistatic scattering measurement is a difficult task since in moving the receiving antenna the phase coherence and the polarization reference coordinates may be perturbed. To avoid these problems we have recently developed a novel method for measuring the bistatic scattering response of a target using a monostatic radar [9]. In this bistatic measurement technique, a wide-band (high-spatial resolution) polarimetric monostatic radar in conjunction with a rotatable perfectly conducting plane positioned behind the target is used to measure the bistatic scattering amplitudes of the target [see Fig. 1(b)]. It is shown that the bistatic scattering matrix of point targets can be measured very accurately while eliminating the complexity of the traditional measurement setup (two antenna system) and its complex calibration procedure. The role of the ground plane is to reflect the desired bistatic response back to the monostatic radar. Primarily, there are three major scattering components that contribute to the signal received by the radar. These scattered signals arrive at the antenna with different delay times. The first component is the direct backscatter from the target which arrives at the antenna with a delay time of

$$
E=\sum_{l=1}^{M} \sum_{i=1}^{N}\left[\left|\frac{s_{v v}^{m}(i, l)-s_{v v}^{t}(i, l)}{s_{v v}^{m}(i, l)}\right|^{2}+\left|\frac{s_{h h}^{m}(i, l) e^{j\left[\phi_{h h_{t}}^{m}(i, l)-\phi_{v v}^{m}(i, l)\right]}-s_{h h}^{t}(i, l) e^{j\left[\phi_{h h l}^{t}(i, l)-\phi_{v v}^{t}(i, l)\right]}}{s_{h h}^{m}(i, l) e^{j\left[\phi_{h h_{l}}^{m}(i, l)-\phi_{v v}^{m}(i, l)\right]}}\right|^{2}\right]
$$


$2 r_{1} / c$, where $c$ is the speed of light. The second component is comprised of two identical subcomponents representing the desired bistatic scattering signals [the solid and dotted lines in Fig. 1(b)]. These two subcomponents arrive at the antenna with a delay time of $\left(r_{1}+r_{2}+r_{3}\right) / c$. The third component is the indirect backscatter of the target in response to the reflected wave from the ground plane. The third component arrives at the antenna with a delay time of $2\left(r_{2}+r_{3}\right) / c$. By choosing the distance between the target and the ground plane to be larger than the radar resolution, all components of the measured backscattered signal can be resolved and collected separately. The reader is referred to [9] for further details on the measurement technique and its corresponding calibration procedure.

In principle, a cluster of particles confined in any geometry of arbitrary boundary can be used so long as the bistatic scattering response of an equivalent homogeneous dielectric material having the same boundary can be evaluated. The effective dielectric constant $\left(\epsilon_{\mathrm{eff}}=\epsilon^{\prime}+j \epsilon^{\prime \prime}\right)$ or the effective index of refraction $\left(n_{\text {eff }}=n^{\prime}+j n^{\prime \prime}\right)$ can be equivalently used for a random media with the effective propagation constant $K=K^{\prime}+j K^{\prime \prime}$ where the equivalent relation is given by $K=k_{o} n_{\mathrm{eff}}, K=k_{o} \sqrt{\epsilon_{\mathrm{eff}}}$. The measured mean bistatic scattering response of the cluster is a function of the equivalent dielectric constant of the dense random media and the shape and size of the enclosing volume. In this paper, a spherical boundary is used since an exact solution (Mie solution) for the bistatic scattered fields of a homogeneous dielectric sphere of arbitrary size can easily be computed [10], [11]. In addition, the symmetry in the spherical geometry eliminates errors due to target misalignment and allows for collecting independent samples simply by rotating the spherical enclosure.

In summary, the measurement procedure involves three steps starting with positioning a cluster of constituent particles of a random medium confined in a spherical volume in front of the ground plane at an appropriate distance. Next, the ground plane is rotated to the desired bistatic angle and the bistatic component of the backscattered signal is gated and measured. Then, the coherent and incoherent components of the scattered fields are separated using many independent measurements of the cluster for a given incident and bistatic directions.

\section{A. Remarks on the Size of Spherical Enclosure}

As was mentioned before, measuring the scattering properties of the random medium can be conducted much more easily when a sample of the random medium is confined into a relatively small spherical geometry. However, for accurate measurements of $K$, the size of the enclosing sphere must be chosen so that the confined cluster of particles preserves the scattering statistics of the infinite random medium. There are a number of conflicting criteria that need to be considered in choosing the size of the enclosure. For example, the cluster must include many particles of the random medium so that the effect of multiple scattering between particles is preserved. Furthermore, the cluster must be larger than many field correlation distances in the random medium so that the statistics of the scattered field are preserved. Based

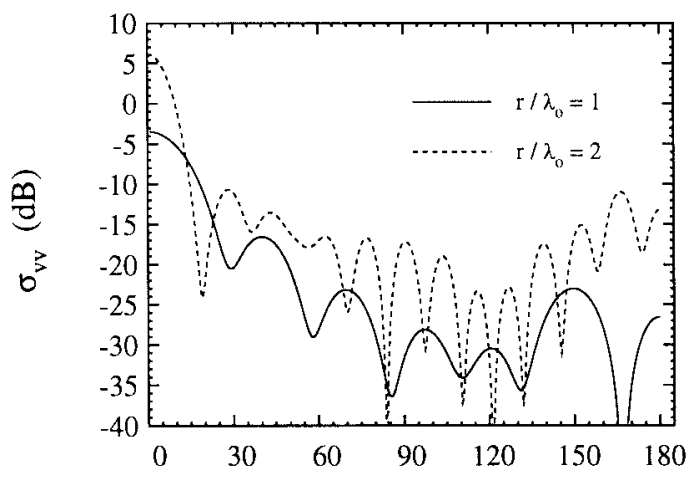

Bistatic Scattering Angle $\theta$ (deg)

Fig. 2. Computed bistatic scattering response of two spheres with $r / \lambda_{o}=1$ and $r / \lambda_{o}=2$ as function of bistatic scattering angle. $n=1.4+j 0.0$ was used in these computations.

on numerical simulations of bistatic scattering from twodimensional (2-D) random media, Sarabandi and Siqueira [12] found that a cluster size as small as one wavelength is enough to obtain the correct estimate of the effective propagation constant when particle sizes are on the order of $\lambda / 10$. On the other hand, a cluster of many wavelengths in size would have a mean bistatic response that would vary rapidly as a function of the bistatic scattering angle (multiple nulls in the pattern). Hence, the measured bistatic response from a large cluster is prone to errors in the rotation angle of the ground plane. Furthermore, it was shown that in the backscattering direction, the standard deviation of the magnitude of the scattered field increased with increasing the size of the cluster [12]. In this case, a large number of independent measurements of the cluster is needed for accurate estimation of the mean backscatter field.

\section{B. Sensitivity Analysis}

Scattering from a homogeneous dielectric sphere is a function of two parameters, namely, the index of refraction $(n=$ $\left.n^{\prime}+j n^{\prime \prime}\right)$ and the normalized radius $r / \lambda_{o}$, where $r$ is the radius of the sphere and $\lambda_{\circ}$ is the wavelength in free-space. In what follows, the sensitivity of the bistatic scattered field of a homogeneous sphere to these two parameters is studied. This sensitivity analysis reveals the accuracy with which the effective index of refraction of random media can be measured.

As mentioned, one of the influential parameters in the bistatic response of a dielectric sphere is the radius normalized to wavelength. As an example, Fig. 2 shows the bistatic responses of two spheres with $r / \lambda_{\circ}=1$ and $r / \lambda_{\circ}=2$ and an index of refraction of $n=1.4+j 0.0$ (a typical value for dry snow). Here, $\theta=0^{\circ}$ and $\theta=180^{\circ}$, respectively, refer to the forward scattering and backscattering directions. It is shown that the overall scattering amplitudes and the number of nulls in the scattering pattern increase with increasing $r / \lambda_{o}$. This sensitivity to normalized radius suggests that if the bistatic scattering measurements were to be conducted for spheres with different radii, a much larger set of independent data would be available for the inversion of the index of refraction. Instead of varying the radius, the wavelength can be varied 


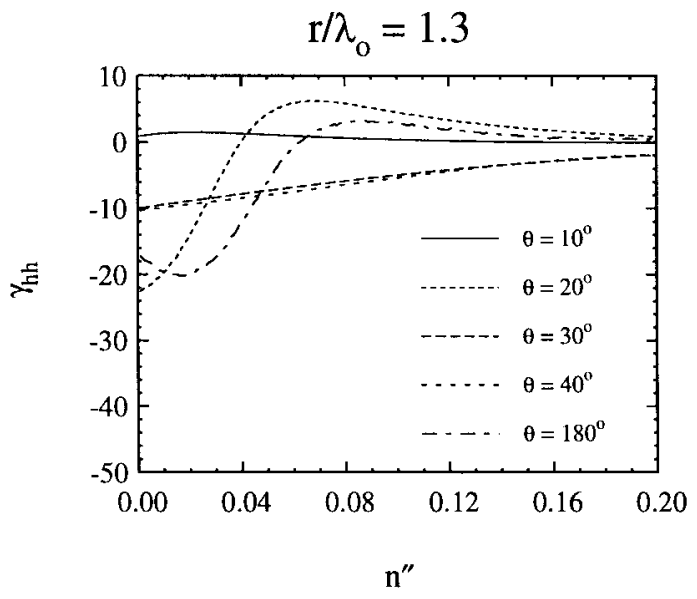

Fig. 3. Computed normalized differential bistatic radar cross section defined by (2) as a function of $n^{\prime \prime}$ for a dielectric sphere with $n^{\prime}=1.4$ and $r / \lambda_{o}=1.3$.

as long as the index of refraction remains constant. This is usually the case when a small bandwidth around the center frequency is used $\left(\Delta f / f_{o}<10 \%\right)$. This can be particularly useful in cases where the size of the spherical enclosure is on the order of many wavelengths and the bistatic response can be measured accurately at a limited number of bistatic angles close to forward scattering direction. In this investigation the frequency response of the bistatic scattering cross section of clusters of random media is measured and used in the inversion algorithm.

For most practical cases of interest, the imaginary part of $K$, which is proportional to the extinction in the random media, is much smaller than the real part. Hence, it is important to determine whether the measurement technique is accurate enough to detect small variations in the imaginary part of the effective index of refraction of the medium. In order to examine the sensitivity of the bistatic scattering radar cross section of a homogeneous sphere $\left(\sigma_{i i}\left(n^{\prime}, n^{\prime \prime}, \theta\right)\right)$ to variations in the imaginary part of the index of refraction, let us define the normalized differential bistatic radar cross section by

$$
\gamma_{i i}(\theta)=\frac{1}{\sigma_{i i}\left(n^{\prime}, n^{\prime \prime}, \theta\right)} \frac{\partial \sigma_{i i}\left(n^{\prime}, n^{\prime \prime}, \theta\right)}{\partial n^{\prime \prime}} ; \quad i=v \text { or } h .
$$

The normalized differential bistatic radar cross section of a dielectric sphere with $n^{\prime}=1.4$ and $r / \lambda_{o}=1.23$ is shown in Fig. 3 as a function of $n^{\prime \prime}$. Fig. 3 indicates that the bistatic scattering cross sections are sensitive to slight changes in $n^{\prime \prime}$ especially when $n^{\prime \prime}$ is small. Therefore, a combination of bistatic scattering response in both frequency and scattering angle should drastically enhance the accuracy in the measurement of $n^{\prime \prime}$.

\section{VerificATION OF THE NeW TECHNIQUE}

In this section, the validity and accuracy of the proposed method is examined by conducting numerical simulations and experiments in an anechoic chamber. For all measurements reported in this paper, a network analyzer-based polarimetric monostatic radar [9] operating at $9.5 \mathrm{GHz}$ with a $1.5-\mathrm{GHz}$ bandwidth was used. The spatial resolution of this system is about $10 \mathrm{~cm}$. A perfectly conducting circular disc with diameter of $1.2 \mathrm{~m}$ was used as the ground plane. The ground plane was positioned inside the anechoic chamber at a distance of $r_{o}=15 \mathrm{~m}$ away from the radar system and the target was located $0.6 \mathrm{~m}$ away from the ground plane, as depicted in Fig. 1(b). The orientation of the ground plane with respect to the radar polarization coordinates was accomplished using a laser system and by maximizing the ground plane's radar return at normal incidence. Precise rotation of the ground plane was facilitated using a computer-controlled stepper motor. The same experimental setup and monostatic radar were used in [9] to demonstrate the accuracy of the bistatic scattering measurement technique. In [9], it is shown that the bistatic scattering cross sections and the phase differences between the copolarized amplitudes of point targets can be measured with an accuracy of $\pm 0.5 \mathrm{~dB}$ and $\pm 5^{\circ}$, respectively.

To examine the accuracy in the measurement of the effective propagation constants of random media using the proposed method, a random medium with low fractional volume is considered. It is well known that the effective field approximation (EFA) method can accurately predict the effective propagation constant of random media in the limiting case of low-volume fractions [6]. Since at low particle densities, the effect of multiple scattering between particles on the overall scattered field is negligible, a simple Monte Carlo simulation based on single scattering properties of particles confined within a finite volume can be performed to demonstrate the feasibility of extracting the effective index of refraction from the mean bistatic pattern of a cluster of particles. In this paper, a random medium with fractional volume $1 \%$ comprised of metallic spheres with diameter $6.35 \mathrm{~mm}$ was chosen as a test case. The metallic spheres (scatterers) were confined within a 7.62 cm spherical boundary $(\sim 2 \lambda$ at $9.5 \mathrm{GHz})$. In such spherical enclosure, on average, only 16 spheres can be enclosed for achieving $1 \%$ fractional volume. The positions of the scatterers inside the spherical enclosure were determined using a random number generator with a uniform distribution. The bistatic scattered field from a spherical cluster of the test medium was computed from coherent addition of scattering from individual particles within the cluster. Overall, the bistatic scattered fields from 1000 independent realizations of the spherical cluster of the test medium were computed in order to evaluate the desired statistics of the bistatic response. Using (1), the effective propagation constant of the test medium calculated from the numerically generated bistatic response is $K_{\text {num }}=(1.0099+$ $j 0.0028) k_{o}$. The effective propagation constant of the test medium under the effective field approximation is $K_{\mathrm{EFA}}=$ $(1.0098+j 0.0033) k_{o}$. It is shown that the result based on the Monte Carlo simulation is in good agreement with EFA.

With confidence in the feasibility of the procedure we proceeded with the experimental verification. For this purpose, ten different spherical clusters of the test medium were constructed where styrofoam spheres with diameter $7.62 \mathrm{~cm}$ were used as hosts for the metallic spheres. The positions of the metallic spheres inside the styrofoam spheres were determined using the random number generator described before. In backscatter, the response from a styrofoam sphere is expected to be 


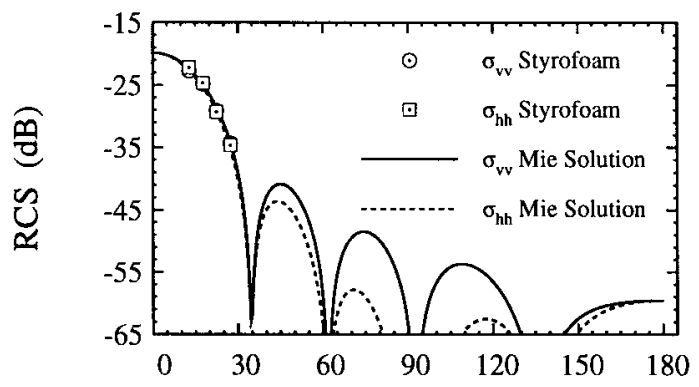

Bistatic Scattering Angle $\theta$ (deg)

Fig. 4. Comparison between the measured mean bistatic scattering radar cross section of a styrofoam sphere $(7.62 \mathrm{~cm}$ in diameter) and the calculated bistatic scattering radar cross section of a homogeneous sphere with $\epsilon_{\text {styro }}=1.0397+j 0.0198$.

undetectable at $X$-band and that is the reason for using styrofoam pedestals as target supports. However, around the forward scattering direction, the bistatic response of styrofoam objects is significant and perhaps comparable to that of a sparse random medium. In fact, the effective propagation constant of the test medium as predicted by EFA is smaller than that of a typical styrofoam. Thus, the effect of styrofoam scattering must be removed from the measured bistatic response of the spheres embedded in the styrofoam sphere. The mean bistatic scattering response from a pure styrofoam sphere identical to those used as hosts for the metallic spheres, was measured using the proposed CBSM technique and is shown in Fig. 4. The measured effective propagation constant of styrofoam was found to be $K_{\text {styro }}=(1.0197+j 0.0097) k_{o}$, which corresponds to $\epsilon_{\text {styro }}=1.0397+j 0.0198$. Then, bistatic measurements were conducted on each of the ten spherical clusters (styrofoam + metallic spheres). Additional independent measurements were obtained by rotating each spherical cluster ten times. Overall, 100 independent measurements were collected at each bistatic angle. The measured average incoherent power of the bistatic scattered field is shown in Fig. 5 and compared with those obtained from the numerical simulation. Then the bistatic scattered fields of the styrofoam sphere were subtracted coherently from the mean bistatic scattered fields of the sphere embedded styrofoam sphere (styrofoam + metallic spheres). The remainder represents the mean bistatic scattered fields of the test medium (metallic spheres). These quantities were then used in (1) to evaluate the effective propagation constant $K_{\text {measr }}$ of the test medium. The measured effective propagation constant of the test medium is $K_{\text {measr }}=(1.0099+j 0.003) k_{o}$ and shows a very good agreement with the effective propagation constant estimated by EFA. The bistatic scattered responses of the styrofoam sphere and the sphere-embedded styrofoam sphere and the subtracted bistatic response along with the best Mie solution fit are shown in Fig. 6 as function of the bistatic scattering angle.

\section{EXPERIMENTAL STUDY OF THE EFFECTIVE PRopagation Constant of Dense Random Media}

In this section, the effects of various electrical and physical properties of dense random media on its effective propagation

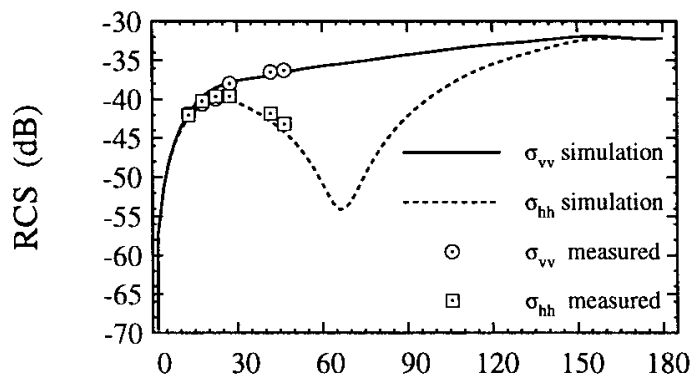

Bistatic Scattering Angle $\theta$ (deg)

Fig. 5. Comparison between the measured and simulated average incoherent bistatic radar cross section of the test medium.

constant are investigated experimentally. In general, the effective propagation constant of a random medium is influenced by the size, shape, orientation distribution, volume fraction, particle arrangement, and permittivity of the constituent particles. The measurements reported in this paper were conducted with the following objectives in mind: 1) examination of the dependence of $K$ on the physical and electrical parameters; 2 ) characterization of the scattering mechanisms in random media as a function of particles' volume fractions; and 3) generating a database as a benchmark for further comparisons with existing theoretical models.

In this paper, random media with spherical particles of fixed size and dielectric constant are considered. As a result, only the effects of the volume fraction, particle arrangement, and the dielectric constant of the constituent particles on the effective propagation constant are studied. Teflon spheres of diameter $6.35 \mathrm{~mm}\left(a / \lambda_{0}=0.1\right.$ at $\left.9.5 \mathrm{GHz}\right)$ and soda-lime glass spheres of diameter $6.0 \mathrm{~mm}\left(a / \lambda_{o}=0.095\right.$ at $\left.9.5 \mathrm{GHz}\right)$ were used to construct random media over a wide range of volume fractions (10-60\%). Permittivity of the Teflon and glass particles were characterized experimentally by measuring the bistatic scattering response of solid Teflon and glass spheres. The dielectric constants of glass and Teflon were found to be $\epsilon_{\text {glass }}=6.93+j 0.10$ and $\epsilon_{\text {tef }}=2.10+j 0.001$, respectively. The large contrast between permittivity of the constituent particles are chosen to demonstrate the effect of multiple scattering on the effective propagation constant. In evaluation of $K$ of constructed dense random media, the copolarized responses ( $V V$ and $H H$ ) of 100 independent spherical clusters of the medium were measured at six bistatic scattering angles $\left(10,20,30,40,50\right.$, and $\left.180^{\circ}\right)$ and 18 frequency points within $0.5 \mathrm{GHz}$ bandwidth centered around 9.5 GHz.

Different methods were used to construct random media with different volume fractions. To construct the $10 \%$ volume fraction, a styrofoam sphere was used as a host for the scattering particles. In this case, similar to the case of $1 \%$ volume fraction the location of particles were determined using the random number generator described before. For higher volume fractions, a hollow styrofoam shell with spherical inner and outer surfaces was used to hold the random cluster of the constituent particles. The inner and outer diameters of the styrofoam shell were $7.62 \mathrm{~cm}\left(r / \lambda_{o}=1.21\right.$ at $\left.9.5 \mathrm{GHz}\right)$ 


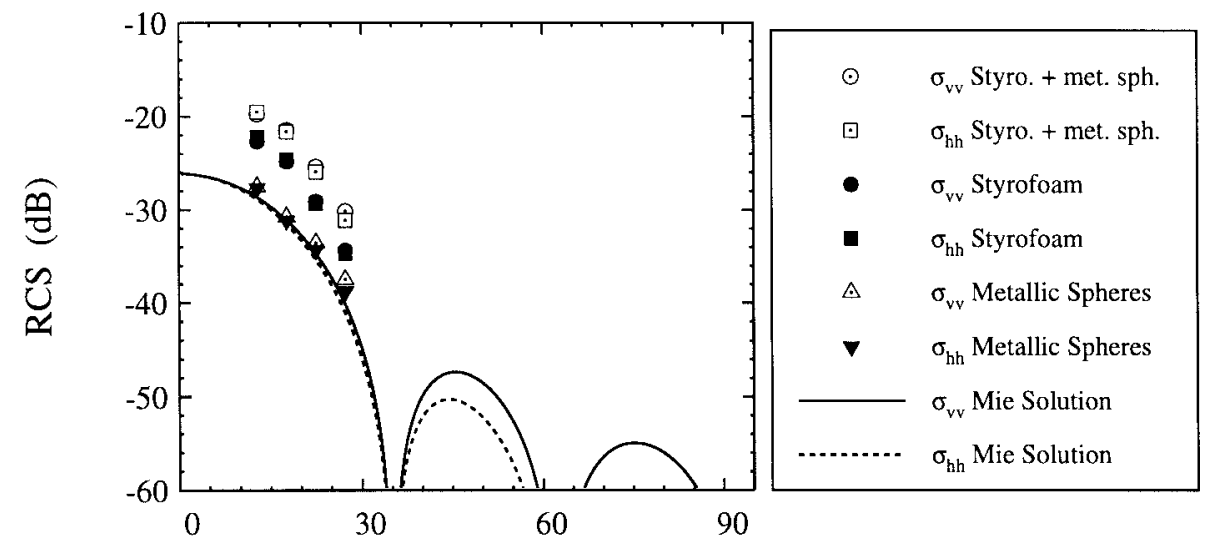

Bistatic Scattering Angle $\theta$ (deg)

Fig. 6. Comparison between the measured bistatic responses of the styrofoam sphere, the sphere embedded styrofoam sphere and the coherently subtracted bistatic scattering response (metallic spheres) along with the best Mie solution fit.

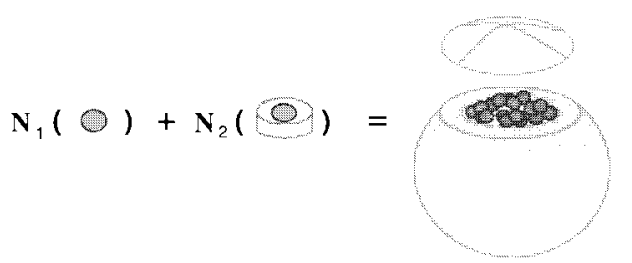

Fig. 7. The technique used for constructing random media with different volume fractions. Appropriate numbers of free and spaced particles are poured into the hollow styrofoam shell.

TABLE I

Average Number of Free and Spaced Glass Particles Required to Fill the Styrofoam Shell $(r=3.81 \mathrm{~cm})$ For Different Volume Fractions

\begin{tabular}{c||c|c|c|c|c}
\hline volume fraction & $20 \%$ & $30 \%$ & $40 \%$ & $50 \%$ & $60 \%$ \\
\hline$N_{1}$ & 170 & 431 & 697 & 962 & 1240 \\
\hline$N_{2}$ & 240 & 183 & 123 & 64 & 0 \\
\hline
\end{tabular}

and $10.16 \mathrm{~cm}$, respectively. In order to achieve the desired volume fraction $(>10 \%)$, a mixture of particles embedded into styrofoam disks (spaced particles) and free particles was poured into the styrofoam shell as shown in Fig. 7. The styrofoam disks were $1.0 \mathrm{~cm}$ in diameter and $0.6 \mathrm{~cm}$ in thickness. The average number of free $\left(N_{1}\right)$ and spaced $\left(N_{2}\right)$ particles required to fill the styrofoam shell are listed in Table I for five different volume fractions used in this experiment. The number of spaced particles was chosen such that the spherical shell would be fully packed with the mixture of free and spaced particles.

Independent realizations of the dense random medium were generated by rotating the cluster of particles in all directions in increments of $20^{\circ}$. In general, the incoherent component of the bistatic scattered field for any realization must be mutually uncorrelated with that of other realizations. To examine whether independent measurements can be realized by simply rotating the cluster, the cross correlation between the incoherent components of the bistatic field as a function of rotation angle (realization number) must be computed. As an example, the normalized correlation function of the bistatic scattered incoherent power of the $40 \%$ packed glass particles, measured at $\theta=20^{\circ}$, is plotted in Fig. 8(a). Fig. 8(a) clearly shows that rotating the cluster of particles in the prescribed manner produces an independent realization of the random medium. It is well known that the statistics of the magnitude of the scattered field from a statistically homogeneous random medium can be described by the Rayleigh probability density function, provided that the medium includes a sufficiently large number of independent scattering centers [13]. Fig. 8(b) shows the histogram of the magnitude of the incoherent component of the bistatic scattered field of the $40 \%$ packed glass particles. In this figure, a Rayleigh probability distribution function (pdf) with the same mean as the histogram is also shown. The Rayleigh pdf agrees well with the histogram of the measured scattered field which implies that the measured samples of dense media were large enough to include many independent scattering centers. The incoherent bistatic RCS of the cluster is the second moment of the bistatic scattered field which represents the fluctuations around the mean bistatic scattered field and can be used to determine the uncertainty in the evaluation of the mean field.

Search routines for the minimization of the nonlinear error function given by (1) may arrive at an incorrect value for $K$ unless certain physical constraints on values of $K$ are imposed. In particular, it is required that $n_{\mathrm{eff}}^{\prime}=K^{\prime} / k_{o} \geq 1$ and $n_{\text {eff }}^{\prime \prime}=K^{\prime \prime} / k_{o} \geq 0$, which set the lower limits of the search routine. An appropriate selection of the upper limit for $n_{\text {eff }}^{\prime}$ is the index of refraction of the constituent particles. Another important issue is an appropriate choice of the initial guess for the search routine. As will be shown later, the Polder-Van Santen mixing formula provides an accurate estimate of the real part of the effective index of refraction of the random medium regardless of the volume fraction. Therefore, the Polder-Van Santen mixing formula is used to provide the initial guess in the search routine. Using the constrained search algorithm, the effective indexes of refraction of the 


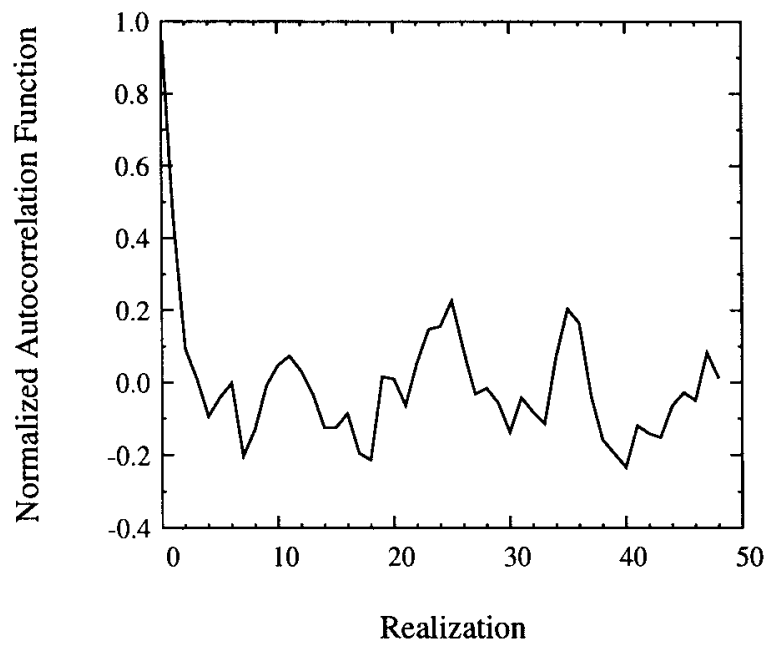

(a)

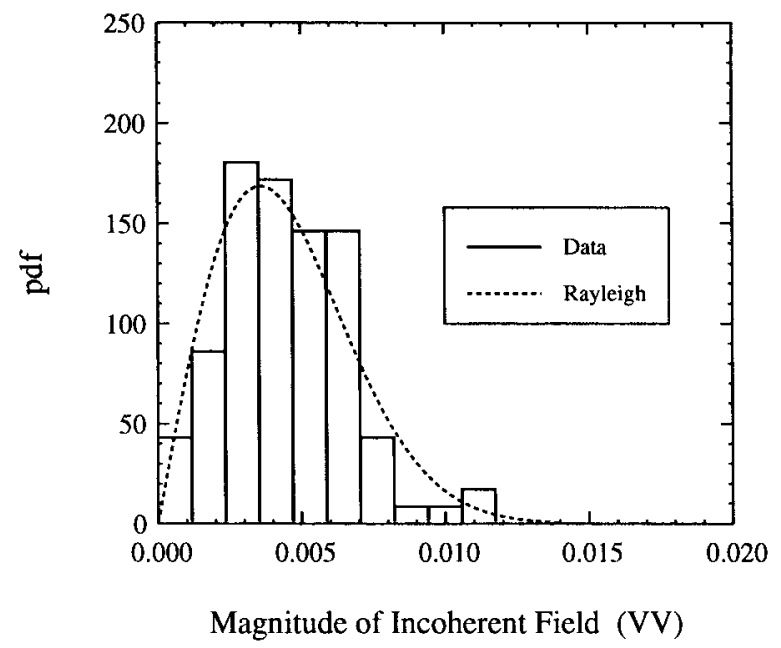

(b)

Fig. 8. (a) The normalized correlation function of the bistatic scattered incoherent power of the $40 \%$ packed glass particles, measured at $\theta=20^{\circ}$, as a function of the realization number. (b) Histogram of the magnitude of the measured incoherent bistatic scattered field of the same random media.

aforementioned random media were accurately characterized. Fig. 9(a) and (b) demonstrates the magnitude and phase of the bistatic scattered field of the random medium constructed from glass spheres with $40 \%$ volume fraction and a homogeneous sphere with $n_{\text {eff }}=1.6457+j 0.0704$ obtained from the search routine. A similar result is shown in Fig. 10 where the bistatic RCS of the random medium constructed from Teflon spheres with $10 \%$ volume fraction and the equivalent homogeneous sphere with $n_{\mathrm{eff}}=1.04736+j 0.00155$ as function of frequency for five bistatic angles are shown. These figures demonstrate the accuracy of the search routine in obtaining the effective index of refraction of the random media used in these experiments.

The measured effective index of refraction $\left(n_{\mathrm{eff}}=K / k_{o}\right)$ for the various dense random media considered in this paper, are summarized in Figs. 11 and 12. In both figures, the measured $n_{\text {eff }}$ are compared with theoretical predictions based on EFA, QCA under the Percus-Yevick pair distribution

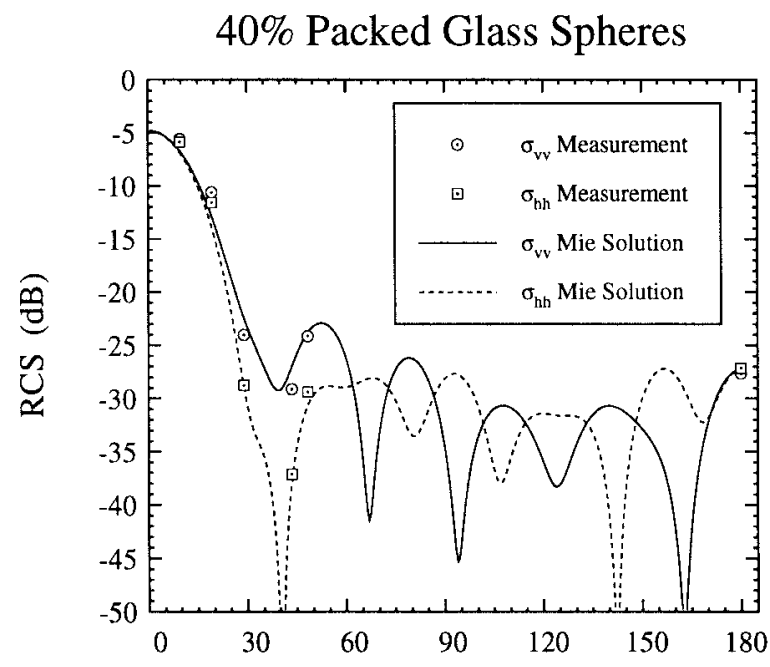

Bistatic Scattering Angle (deg)

(a)

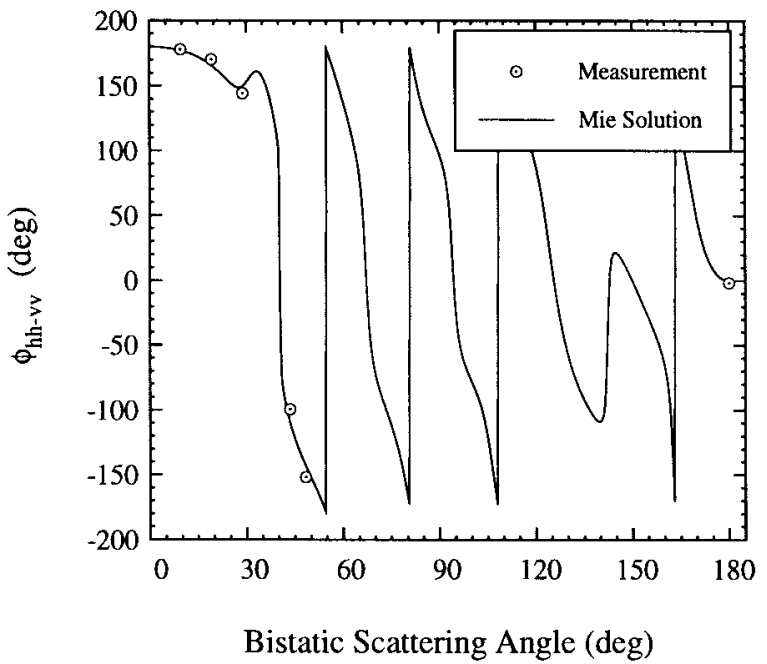

(b)

Fig. 9. The measured mean bistatic radar cross section and the copolarized phase difference of $40 \%$ packed glass particles are compared with the theoretical responses of a homogeneous sphere $(7.62 \mathrm{~cm}$ in diameter) computed at $9.5 \mathrm{GHz}$ with $n_{\mathrm{eff}}=1.6457+j 0.0704$ derived from the inversion algorithm.

function (QCA-PY), and Polder-Van Santen mixing formula as functions of the volume fraction. It is observed that for both dielectric materials, the mixing formula can reasonably estimate the real part of $n_{\mathrm{eff}}$. None of the discussed models and, in particular the QCA-PY, were able to predict correctly the imaginary part of $n_{\text {eff }}$ for volume fractions above $10 \%$. Comparing Figs. 11(b) and 12(b), it can be seen that the scattering losses in media constructed from glass spheres are much higher than those of the media constructed from Teflon spheres. This is due to stronger scattered fields generated by the glass spheres with higher permittivity contrast than the Teflon spheres.

As the volume fraction of a random medium increases, it is expected that the internal fields of the scatterers within the random medium become correlated and, therefore, the 


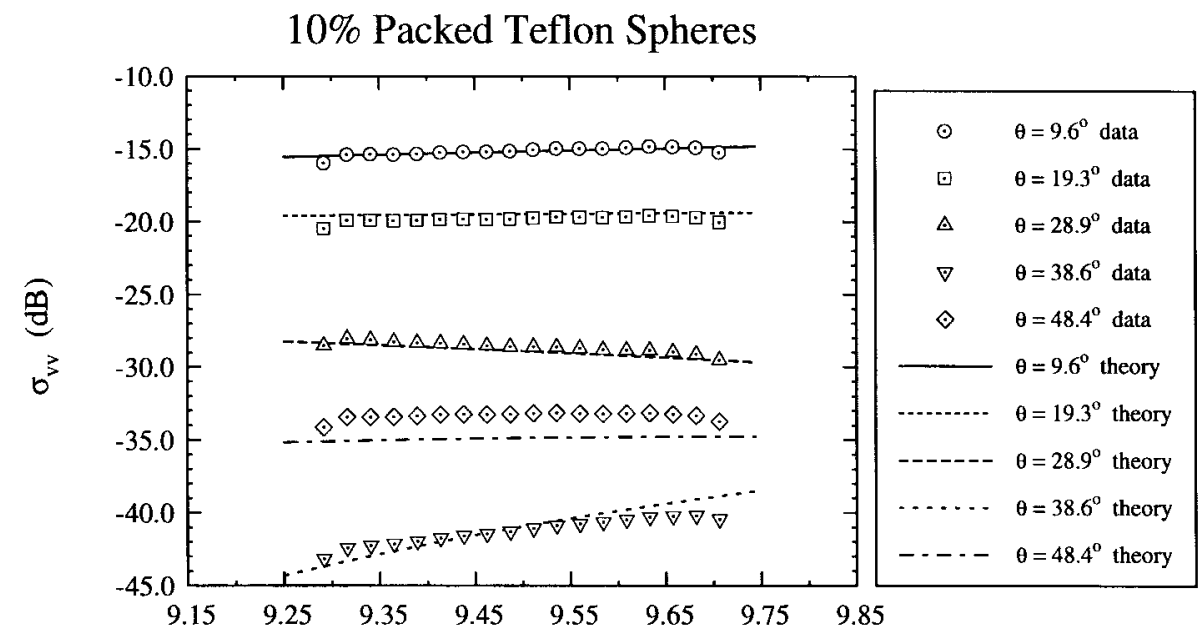

Frequency $(\mathrm{GHz})$

Fig. 10. The measured mean bistatic radar cross section of $10 \%$ packed Teflon particles, as function of frequency, is compared with the theoretical response of a homogeneous sphere with $n_{\mathrm{eff}}=1.04736+j 0.00155$ derived from the inversion algorithm.

Teflon

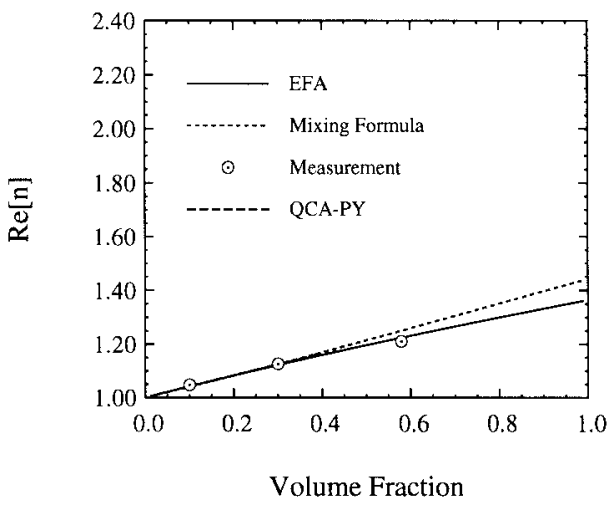

(a)

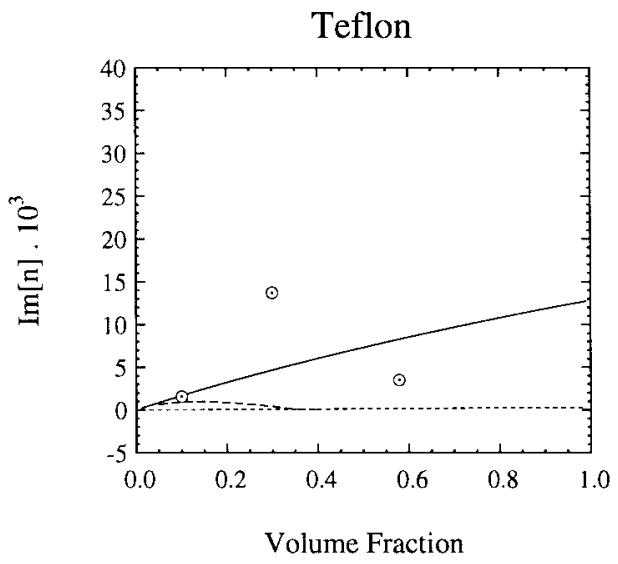

(b)

Fig. 11. The effective index of refraction (a) real part and (b) imaginary part of random media constructed from Teflon particles as function of volume fraction. The measured effective index of refraction is compared with those computed using EFA, QCA-PY, and Polder-Van Santen mixing formulas.

incoherent scattered field be reduced. Based on this argument, one may deduce that the scattering loss predicted by the EFA is an upper limit. However, as our experimental results indicate [see Figs. 11(b) and 12(b)], this is not the case. At intermediate

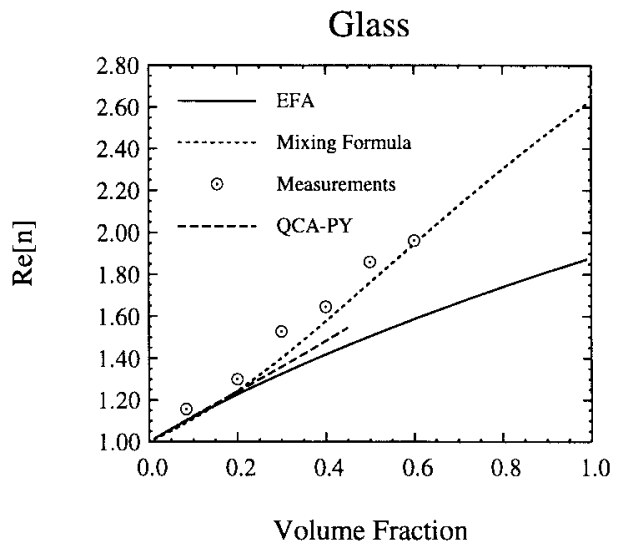

(a)

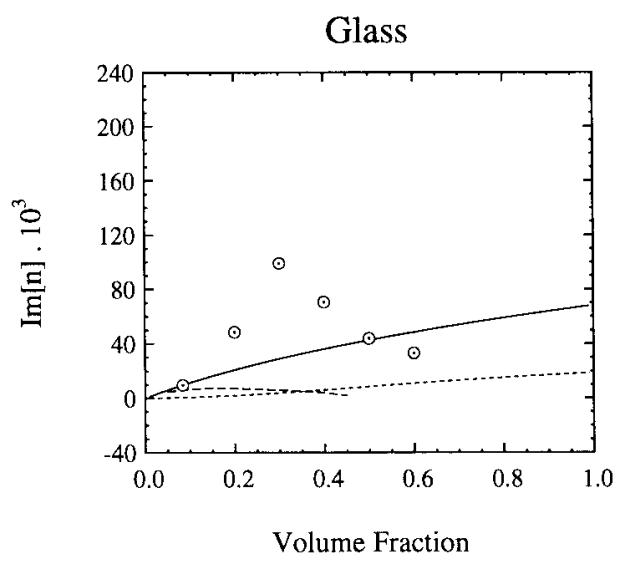

(b)

Fig. 12. The effective index of refraction (a) real part and (b) imaginary part of random media constructed from glass particles as function of volume fraction. The measured effective index of refraction is compared with those computed using EFA, QCA-PY, and Polder-Van Santen mixing formulas.

volume fractions (15-50\%) the scattering loss in both glass and Teflon media is more than what is predicted by the EFA. This phenomenon can be explained by examining the particle arrangement at these intermediate volume fractions. In these cases, the probability of finding two or more connected par- 
ticles is rather high. The connected particles may be viewed as a larger particle whose scattered field is much higher than the sum of the scattered field from isolated particles which in turn produces higher incoherent scattered power in the random media.

\section{CONCLUSION}

A new technique for measuring the effective propagation constant of dense random media is presented. In this technique, the mean bistatic scattered fields of a cluster of random medium, confined in a known geometrical boundary, are measured using a monostatic radar and a rotatable ground plane. Then the measured mean bistatic scattered fields are fitted to the bistatic scattered fields of a homogeneous lossy material with the same geometrical boundary. The accuracy of the new technique in measuring the effective propagation constant of a dense random medium was verified both experimentally and numerically in the low volume fraction limiting case. It is also shown that using this technique, the permittivity of low dielectric materials such as styrofoam can be measured very accurately. Measurements of the effective propagation constants of different dense random media comprised of homogeneous spherical particles with different packing densities are reported and compared with existing analytical models. It is shown that none of the existing analytical models are able to predict the extinction accurately at volume fractions beyond $10 \%$. It is also shown that the Polder-Van Santen mixing formula can be used to predict the real part of $K$ with reasonable accuracy.

\section{REFERENCES}

[1] F. T. Ulaby, R. K. Moore, and A. K. Fung, Microwave Remote Sensing: Active and Passive. Norwood, MA: Artech House, 1986, vol. 3.

[2] L. Tsang, J. A. Kong, and R. T. Shin, Theory of Microwave Remote Sensing. New York: Wiley, 1985.

[3] J. R. Kendra, F. T. Ulaby, and K. Sarabandi, "Snow probe for in situ determination of wetness and density," IEEE Trans. Geosci. Remote Sensing, vol. 32, pp. 1152-1159, Nov. 1994.
[4] K. Sarabandi and E. S. Li, "A microstrip ring resonator for soil moisture measurements," IEEE Trans. Geosci. Remote Sensing, vol. 35, pp. 1223-1231, Sept. 1997.

[5] E. Nyfors and P. Vainikainen, Industrial Microwave Sensors. Norwood, MA: Artech House, 1989.

[6] C. E. Mandt, Y. Kuga, L. Tsang, and A. Ishimaru, "Microwave propagation and scattering in a dense distribution of nontenuous spheres: Experiment and theory," Waves in Random Media, vol. 2, pp. 225-234, 1992.

[7] M. T. Hallikainen, F. T. Ulaby, and M. Abdelrazik, "Dielectric properties of snow in the 3-37 GHz range," IEEE Trans. Antennas Propagat., vol. AP-34, pp. 1329-1340, Nov. 1986.

[8] J. L. Zhou and A. L. Tits, "User's guide for FSQP Version 3.3b: A FORTRAN code for solving constrained nonlinear (minimax) optimization problems, generating iterates satisfying all inequality and linear contraints,” Elect. Eng. Dept., Inst. Syst. Res., Univ. Maryland, College Park, MD, 1993.

[9] K. Sarabandi and A. Nashashibi, "A novel bistatic scattering matrix measurement technique using a monostatic radar," IEEE Trans. Antennas Propagat., vol. 44, pp. 41-50, Jan. 1996.

[10] G. T. Ruck, D. E. Barrick, W. D. Stuart, and C. K. Krichbaum, Radar Cross Section Handbook. New York: Plenum, 1970.

[11] C. F. Bohren and D. R. Huffman, Absorption and Scattering of Light by Small Particles. New York: Wiley, 1983.

[12] K. Sarabandi and P. Siqueira, "Numerical scattering analysis for two dimensional dense random media: Characterization of effective permittivity," IEEE Trans. Antennas Propagat., vol. 45, pp. 858-867, May 1997.

[13] K. Sarabandi, "Derivation of phase statistics from the Mueller matrix," Radio Sci., vol. 27, no. 5, pp. 553-560, Sept./Oct. 1992.

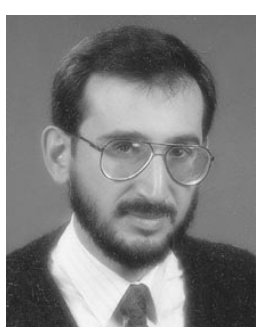

Adib Nashashibi (S'82-M'95) received the B.Sc. and M.Sc. degrees in electrical engineering from Kuwait University, Kuwait City, Kuwait, in 1985 and 1988, respectively, and the Ph.D. degree in electrical engineering from the University of Michigan, Ann Arbor, in 1995.

He is currently an Assistant Research Scientist at the Radiation Laboratory at the University of Michigan. His research interests include microwave remote sensing, polarimetric millimeter-wave radars, calibration and measurement techniques, electromagnetic wave propagation, and scattering in random media.

Kamal Sarabandi (S'87-M'90-SM'92), for a photograph and biography, see p. 861 of the May 1999 issue of this Transactions. 